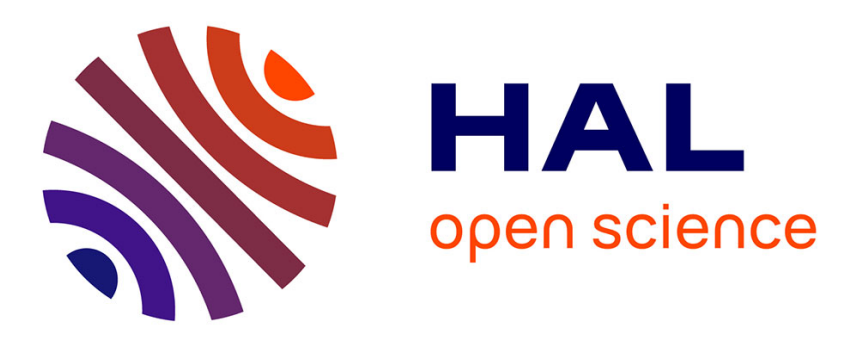

\title{
Some applications of nanometer scale structures for current and future X-ray space research
}

F. Christensen, S. Abdali, P. Frederiksen, A. Hornstrup, I. Rasmussen, N. Westergaard, H. Schnopper, E. Louis, H.-J. Voorma, N. Koster, et al.

\section{- To cite this version:}

F. Christensen, S. Abdali, P. Frederiksen, A. Hornstrup, I. Rasmussen, et al.. Some applications of nanometer scale structures for current and future X-ray space research. Journal de Physique III, 1994, 4 (9), pp.1599-1612. 10.1051/jp3:1994227 . jpa-00249210

\section{HAL Id: jpa-00249210 https://hal.science/jpa-00249210}

Submitted on 1 Jan 1994

HAL is a multi-disciplinary open access archive for the deposit and dissemination of scientific research documents, whether they are published or not. The documents may come from teaching and research institutions in France or abroad, or from public or private research centers.
L'archive ouverte pluridisciplinaire HAL, est destinée au dépôt et à la diffusion de documents scientifiques de niveau recherche, publiés ou non, émanant des établissements d'enseignement et de recherche français ou étrangers, des laboratoires publics ou privés. 


\title{
Some applications of nanometer scale structures for current and future $X$-ray space research
}

F. E. Christensen ( $\left.{ }^{1}\right)$, S. Abdali ( $\left.{ }^{1}\right)$, P. K. Frederiksen ( $\left.{ }^{(}\right)$, A. Hornstrup ( $\left.{ }^{1}\right)$, I. Rasmussen ( $\left.{ }^{1}\right)$, N. J. Westergaard $\left({ }^{1}\right)$, H. W. Schnopper $\left({ }^{1}\right)$, E. Louis $\left({ }^{2}\right)$, H.-J. Voorma $\left({ }^{2}\right)$, N. Koster $\left({ }^{2}\right)$, H. Wiebicke $\left({ }^{3}\right)$, I. Halm $\left({ }^{3}\right)$, U. Geppert $\left({ }^{3}\right)$, E. Silver $\left({ }^{4}\right)$, M. Legros $\left({ }^{4}\right)$, K. Borozdin $\left({ }^{5}\right)$, K. D. Joensen $\left({ }^{6}\right)$, P. Gorenstein $\left({ }^{6}\right)$, J. Wood $\left({ }^{7}\right)$ and G. Gutman ( $\left.{ }^{7}\right)$

(1) Danish Space Research Institute, Gl. Lundtoftevej 7, DK-2800 Lyngby, Denmark

(') FOM-Institute for Plasma Physics, Rijnhuizen, Edisonbaan 14, NL-3430 BE Nieuwegein. The Netherlands

( $\left.{ }^{3}\right)$ Max-Planck-Institut für Extraterrestrische Physik, Aussenstelle Berlin-Adlershof, Rudower Chaussee 5, 12489 Berlin, Germany

${ }^{4}$ ) Laboratory for Experimental Astrophysics, Lawrence Livermore National Laboratory, P. O. Box 5511, L-637, Livermore, CA 94550, U.S.A.

( $\left.{ }^{5}\right)$ Space Research Institute, Russian Academy of Sciences, High Energy Astrophysics Dept. Profsojusnaja 84/32, 117810 Moscow, Russia

(6) Smithsonian Astrophysical Observatory, 60 Garden Street, Cambridge, MA 02138, U.S.A.

(7) Ovonics Synthetic Materials Company, 1788 Northwood Drive, Troy, Michigan 48084, U.S.A.

(Received 19 November 1993, accepted 3 March 1994)

\begin{abstract}
Nanometer scale structures such as multilayers, gratings and natural crystals are playing an increasing role in spectroscopic applications for $\mathrm{X}$-ray astrophysics. A few examples are briefly described as an introduction to current and planned applications pursued at the Danish Space Research Institute in collaboration with the FOM Institute for Plasma Physics, Nieuwegein, the Max-Planck-Institut für Extraterrestrische Physik, Aussenstelle Berlin, the Space Research Institute, Russian Academy of Sciences, the Smithsonian Astrophysical Observatory, Ovonics Synthetic Materials Company and Lawrence Livermore National Laboratory. These examples include : 1. the application of multilayered Si crystals for simultaneous spectroscopy in two energy bands one centred around the SK-emission near $2.45 \mathrm{keV}$ and the other below the CK absorption edge at $0.284 \mathrm{keV} ; 2$. the use of in-depth graded period multilayer structures for broad band spectroscopy in the energy range up to $100 \mathrm{keV} ; 3$. the potential use of large perfect asymmetrically cut $\mathrm{Si}$ or Ge crystals combined with a short focal length multilayer telescope for ultra high energy resolution solar/stellar spectroscopy with $E / \Delta E>10^{4}$ and : 4. high throughput multilayer coated telescope for high resolution $\mathrm{Fe} \mathrm{K}$ line spectroscopy with microcalorimeters.
\end{abstract}




\section{Introduction.}

Nanostructures have been used extensively in X-ray space research for the past 10-15 years. Natural crystals were used for high resolution solar spectroscopy in instruments such as the bent crystal spectrometer on the solar maximum mission [1]. The focal plane crystal spectrometer on the Einstein observatory (HEAO 2) provided high resolution spectroscopy of intense soft $X$-ray sources [2]. The Einstein observatory was also the first satellite where thin film coating technology was applied to grazing incidence focusing optics [3] for non-solar Xray Astronomy. Einstein and later the first ESA X-ray satellite EXOSAT [4] used transmission gratings. Reflection gratings will be used in the next ESA X-ray satellite XMM [5] and transmission gratings will be used again in the low energy transmission grating spectrometer [6] and the high energy grating spectrometer [7] on the forthcoming NASA X-ray satellite AXAF-I. The first use of multilayers in X-ray space research was pioneered in 1985 in a normal incidence soft $X$-ray telescope flown on a solar rocket [8]. Since then a series of very successful solar rocket flights has taken place utilizing multilayer coated $\mathrm{X}$-ray telescopes operating primarily near $63 \AA[9]$ using $\mathrm{Co} / \mathrm{C}$ multilayers and near $173 \AA$ using Mo/Si multilayers [10]. The use of multilayers in normal incidence telescope programs continues in several projects. Two of these that extend the use of multilayer coatings to cosmic (non-Solar) studies are the ALEXIS mini satellite [11] which is currently flying and EUVITA, an array of normal incidence multilayer telescopes which will fly on the Russian spectrum Röntgen gamma (SRG) mission [12].

The purpose of this paper is to give a review of some novel applications of nanostructures for $\mathrm{X}$-ray space research that we have pursued within the past five years. The first is the combination of natural crystals and multilayers in the objective crystal spectrometer which is to fly on the SRG satellite. The second is the use of in-depth graded period multilayers for a hard $\mathrm{X}$-ray telescope with imaging capabilities up to $100 \mathrm{keV}$. The third is the combination of a short focal length, multilayered telescope with large asymmetrically perfect crystals of Si or Ge for ultra-high resolution spectroscopy $\left(E / \Delta E \geqslant 10^{-4}\right)$ near FeK-emission lines. The last is the combination of a high throughput imaging telescope based on multilayer coatings with high resolution $\left(E / \Delta E=10^{2}-10^{3}\right)$ microcalorimeter arrays for $\mathrm{FeK}$ line spectroscopy.

\section{The objective crystal spectrometer-OXS.}

The concept of an objective crystal spectrometer for X-ray Astronomy was first proposed in 1970 by Angel and Weisskopf [13]. It consists of a panel of crystals in front of an imaging telescope. A detailed study of its capabilities for spectral imaging of extended sources was published by Schnopper and Byrnak in 1987 [14]. The objective crystal spectrometer is included in the payload for the spectrum Röntgen gamma satellite. The basic modes of operation of the spectrometer is described in detail in reference [14] and its most elaborate mode of operation involves scanning the crystal panel in narrow angular ranges for a number (10-20) of reorientations of the telescope. In 1990 Christensen et al. [15] described the basic configuration of the spectrometer to be flown on the spectrum Röntgen gamma satellite and proposed the use of multilayer coatings on the chosen natural crystals to allow simultaneous spectroscopy in two separate wavelength bands. Soft $X$-ray wavelengths are reflected in the multilayer coating and hard X-ray wavelengths are reflected in the parent crystal. The energy resolution of the focal plane detector is used to separate the two energies. Figure 1 shows the principle.

The crystal panel had two sides covered with crystals. One side will be completely covered with $\mathrm{LiF}(220)$ for $\mathrm{FeK}$ line spectroscopy. The dimensions of each crystal is approximately $63 \times 23 \mathrm{~mm}^{2}$ and 314 crystals will be needed to cover the whole panel. The crystals are 


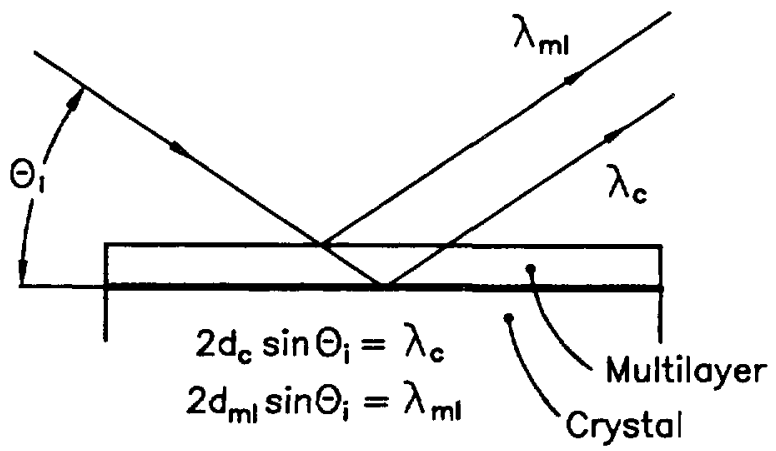

Fig. 1. - The principle of simultaneous spectroscopy in two energy bands using a multilayer coated crystal. Reflection of low energy X-ray line emission takes place in the multulayer stack while the high energy emission line penetrates the multilayer with little or no absorption and is reflected in the natural crystal.

manufactured by Carl Zeiss Jena and provided by the Max-Planck-Institut für Extraterrestrische Physik, Aussenstelle Berlin. We have recently performed a detailed X-ray study of the 441 available flight quality crystals at $\mathrm{CuK}_{\alpha_{1}}[16,17]$ and based on this study 160 crystals were milled on the backside to bring the angle between the backside (gluing side) and the (220)planes within a much narrower tolerance. By selecting the 314 best crystals, the estimated overall rocking curve width (FWHM) for the whole panels is $2.34 \mathrm{arcmin}$ at $\mathrm{CuK}_{\alpha,}$ and the peak reflectivity of the whole panel should be 0.23 . These parameters have also been measured at $\mathrm{FeK}_{\alpha_{1}}$ which is a wavelength close to where the crystals will be used. Using both of these results the rocking curve width (FWHM) is 2.64 arcmin and the peak reflectivity is 0.21 at the appropriate wavelength for He-like Fe. This is a factor of 1.6 times better for the rocking curve width and a factor of 1.7 better for the peak reflectivity compared to what would be obtained without the elaborate measuring program and the careful reprocessing. This result will have a major impact on what can be accomplished with the objective crystal spectrometer. With the optimized parameters given above the energy resolution $E / \Delta E$ is estimated to be 1200 at $\mathrm{FeK}_{\alpha_{1}}(6.404 \mathrm{keV})$.

The other side of the crystal panel will be partly covered with RAP $(001)$ crystals for OK line spectroscopy and $\mathrm{Si}(111)$ crystals for SK spectroscopy. Two thirds of the area will be covered with $240 \mathrm{RAP}(001)$ crystals of dimensions $20 \times 60 \mathrm{~mm}^{2}$ and one third of the area will be covered with $40 \mathrm{Si}(111)$ crystals of dimensions $60 \times 60 \mathrm{~mm}^{2}$. The RAP crystals have been manufactured by X-Ray Optics, Ltd. in St. Petersburg and provided to DSRI by the Space Research Institute in Moscow. The Si(111) crystals are made by Topsil in Denmark and accurately cut and aligned by Eagle Picher Research Laboratories in the USA. They have been polished by Virginia Semiconductor Inc. in the USA. For the RAP crystals an energy resolution of $E / \Delta E>700$ should be obtained, while for the Si crystals the theoretical energy resolution is $>5000$ and will be limited by the accuracy of the cutting and mounting which is as yet undetermined. Our estimate is that $E / \Delta E$ will be $\sim 3200$ at $2.45 \mathrm{keV}$.

Multilayer coating is planned for the 40 polished $\mathrm{Si}(111)$ surfaces and the coating is designed to be used in the energy range below the CK absorption edge of $0.284 \mathrm{keV}$ in the detector window material. The period of the multilayer is chosen to allow $63 \AA$ radiation to be reflected at the same Bragg angle as the Si crystal reflects the center of the He-like $\mathrm{S}$ emission line complex $(5.06 \AA)$. This angle is $53.79^{\circ}$ which then determines that the period of the multilayer should be $39 \AA$. The angular range within which the crystal panel can be rotated 
without significant vignetting together with the loss of transmission in the detector window sets the low energy limit for the use of the multilayer coating. The active range for the multilayer coating is thus from $44 \AA$ to $71 \AA$. The absorption in the multilayer of the $\mathrm{S}$ line emission which is reflected by the $\mathrm{Si}$ crystals can be reduced by choosing either $\mathrm{Ni}, \mathrm{Cr}$ or $\mathrm{Co}$ as the heavy element and $\mathrm{C}$ as the light element. With these combinations the theoretical energy resolution in the energy range given above is $60-90$ which implies that less than 100 periods are needed. The requirement for a minimum thickness of the heavy element to produce a high quality coating and the desire to reduce the absorption of SK wavelength by the heavy material in the multilayer has led to a compromise specification for the thickness of the heavy material of $15 \AA$. Thus, the multilayer is completely specified and at the center wavelength of the $\mathrm{He}$ like $\mathrm{S}$ emission $(5.06 \AA$ ) there is $81 \%$ transmission in a 100 period $\mathrm{Cr} / \mathrm{C}$ multilayer coating (calculated assuming bulk densities of $\mathrm{Cr}$ and $\mathrm{C}$ ). For this $\mathrm{Cr} / \mathrm{C}$ coating, the reflectivity versus angle of incidence for a number of wavelengths in the range from $46 \AA$ to $66 \AA$ has been calculated assuming an interfacial roughness of $4 \AA$ (which is what can be realistically achieved [18]). The results indicate that the peak reflectivity varies slowly from $21 \%$ at $46 \AA$ to $14 \%$ at $66 \AA$, while the energy resolution varies from 74 at $46 \AA$ to 85 at $66 \AA$. To achieve these energy resolutions, the requirement on the constancy of the period of the multilayer coating for all of the deposited area is $1 \%$. The accuracy on the absolute value of the period is $\pm 1 \AA$. The multilayers will be deposited at the FOM Institute for Plasma Physics, Nieuwegein in The Netherlands. This newly established multilayer coating facility uses ion assisted $E$ beam evaporation which has been proved to be very efficient in producing high quality multilayers of the type required in this application [18, 19]. The first depositions using ion etching have been made at the FOM facility where it was demonstrated that $83 \%$ of theoretical reflectivity could be obtained from a Mo/Si multilayer with 32 periods i.e., more than $50 \%$ reflectivity was obtained at $133 \AA$ [20]. In addition it was demonstrated that the combination of ion etching and in-situ X-ray monitoring enables correction of almost any layer thickness error during the deposition process. With the current estimates of reflectivities of the LiF, the RAP, the Si crystals and the multilayers on Si we have calculated the effective area in the four energy windows which are accessible with the objective crystal spectrometer (Fig. 2).

\section{A hard X-ray telescope based on in-depth graded period multilayers-HXT.}

Focusing optics in the 10-100 keV band have not previously been flown and many interesting problems in high energy astrophysics await the launch of such a mission. In recent years there have been several activities which aim at the development of focusing optics in this energy band. Among them are microchannel plates [21], microcapillaries [22] and graphite crystals $[23,24]$. Of these approaches the microchannel plates and the microcapillaries, are necessarily based on multi-focus designs since the reflecting surface is glass which has rather small critical angles for total reflection at energies above $10 \mathrm{keV}$. The microcapillaries can only be used as a concentrator and will not allow imaging. The same is true for the graphite crystal approach. In 1991 Christensen et al. [25] proposed a high energy telescope design which combines grazing incidence geometry with Bragg reflection in an in-depth graded period multilayer coating. This concept combines the desirable imaging properties of a conical approximation to a Wolter 1 [26] telescope and the high reflectivity in the $10-100 \mathrm{keV}$ band that is provided by the graded period multilayer mirrors - also known as X-ray supermirrors. The method of grading the period of multilayers has been used for many years for neutron supermirrors [27], however, the neutron supermirror designs are relatively simple since absorption plays no role. For X-ray supermirrors, absorption must be taken into account leading to different coating designs.

The idea of grading the period of multilayers to create a wide reflection band for X-rays only 


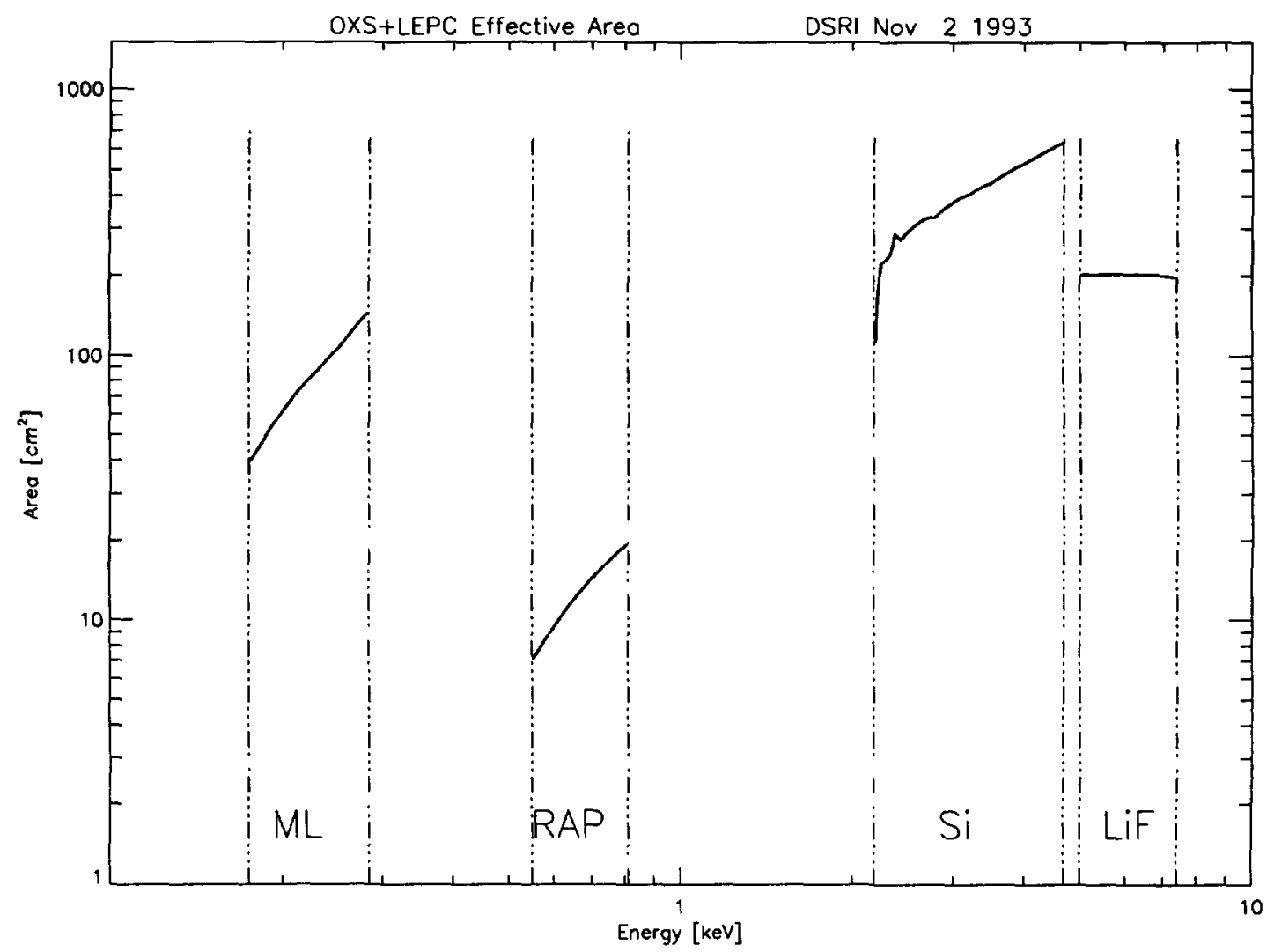

Fig. 2. - The effective area in the four energy windows accessible to the objective crystal spectrometer using a low energy proportional counter (LEPC). There will be considerable effective area outside of these windows depending on the actual geometrical obscurations on the satellite. Si(111) covers both Helike $\mathrm{Ca}(3.886 \mathrm{keV})$, He-like $\operatorname{Ar}(3.131 \mathrm{keV})$ and He-like $\mathrm{S}(2.450 \mathrm{keV})$.

works for hard $\mathrm{X}$-rays $(>10 \mathrm{keV}$ ) where the penetration depth is appreciable. The principle is shown in figure 3 where the harder X-rays are reflected from the bottom of the stack and the softer X-rays are reflected from the top of the stack. The first design for a hard X-ray telescope based on graded period multilayers was used to demonstrate what could be obtained from an existing telescope project namely the SODART telescopes [28] to be launched on the spectrum Röntgen gamma satellite. The Au coating is simply replaced by a supermirror design which aimed at achieving the highest effective area near $40 \mathrm{keV}$. The SODART telescopes are based on tight nesting of conical mirrors in an approximation to a Wolter 1 design [30] and the parameters of the SODART-like model telescope, called MULTI-X, are given in table I.

For the design in table I the on-axis angle of incidence varies from $\sim 0.1$ to $\sim 0.5^{\circ}$. The multilayer coating design is changed at regular intervals from the outermost mirrors to the innermost mirrors in order to generate a specific effective area profile at energies above $\sim 10 \mathrm{keV}$. The multilayer structure versus the on-axis incident angle has been iterated until a maximum effective area around $40 \mathrm{keV}$ was reached. This effect was achieved by using 20 different coatings with total period variations from $25 \AA$ to $100 \AA$ [27]. The shells which mainly reflect $\mathrm{X}$-rays below $20 \mathrm{keV}$ (on-axis) are the outermost ones where the on-axis angle of incidence is greater than $\sim 0.4^{\circ}$ This led to the choice of Mo as the heavy element for these 


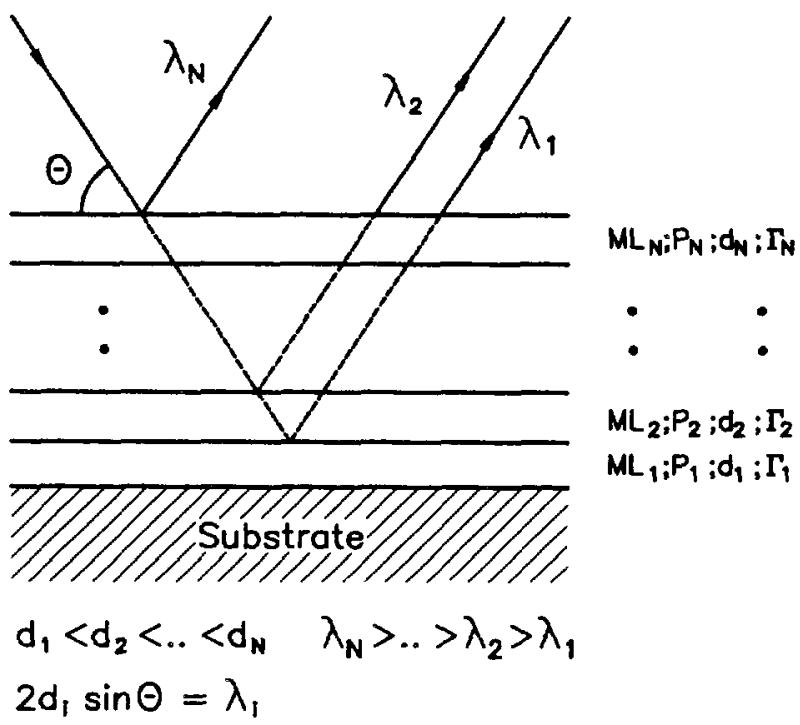

Fig. 3. - An in-depth graded period multilayer structure. The first layers deposited on the substrate is designated $\mathrm{ML}_{1}$ and has $P_{1}$ periods with $d_{1}$ as the period and $\Gamma_{1}$ is the ratio of the thickness of the heavy element to $d_{1}$. The deposition process is continued until there are $N$ different layer sets where $d_{1}<d_{2} \cdot<d_{N}$.

Table I. - Telescope parameters of MULTI-X.

\begin{tabular}{|lc|}
\hline Focal length & $8000 \mathrm{~mm}$ \\
Number of shells & 194 \\
Outer shell diameter & $600 \mathrm{~mm}$ \\
Inner shell diameter & $100 \mathrm{~mm}$ \\
Mirror length & $200 \mathrm{~mm}$ \\
Thickness & $0.3 \mathrm{~mm}$ \\
\hline
\end{tabular}

shells since Mo has a $\mathrm{K}$ absorption edge very close to $20 \mathrm{keV}$. Ni was chosen for the remaining shells. $\mathrm{C}$ was chosen in all cases as the light element (as opposed to Si) to minimize absorption. Figure 4 shows the theoretical on-axis effective area versus energy for this graded period multilayer telescope compared to what may be obtained with the same telescope with Aucoated shells. The calculation is based on perfect multilayer coatings. Since this first attempt at designing a hard X-ray telescope based on graded period multilayers, Joensen et al. [29] have studied the performance of short focal length multi-focus designs that are based on both the conical approximation to a Wolter 1 design and the Kirk-Patrick-Baez design. Tables II and III list the two designs that have been studied and figure 5 gives the effective area. These calculations have also simulated the effect of interfacial roughness (Fig. 5). Figures 4 and 5 clearly demonstrate the potential usefulness of graded period multilayers in X-ray space research and, if these telescopes can be manufactured, exciting results will follow for high energy astrophysics. A number of X-ray supermirrors have been produced at Ovonics Synthetic Materials Co. that contain up to 500 periods of $\mathrm{Ni} / \mathrm{C}$ with periods ranging from $\sim 25 \AA$ to $\sim 100 \AA$. In addition, a W/Si supermirror has been produced and extended 
Eff. Area vs. Energy

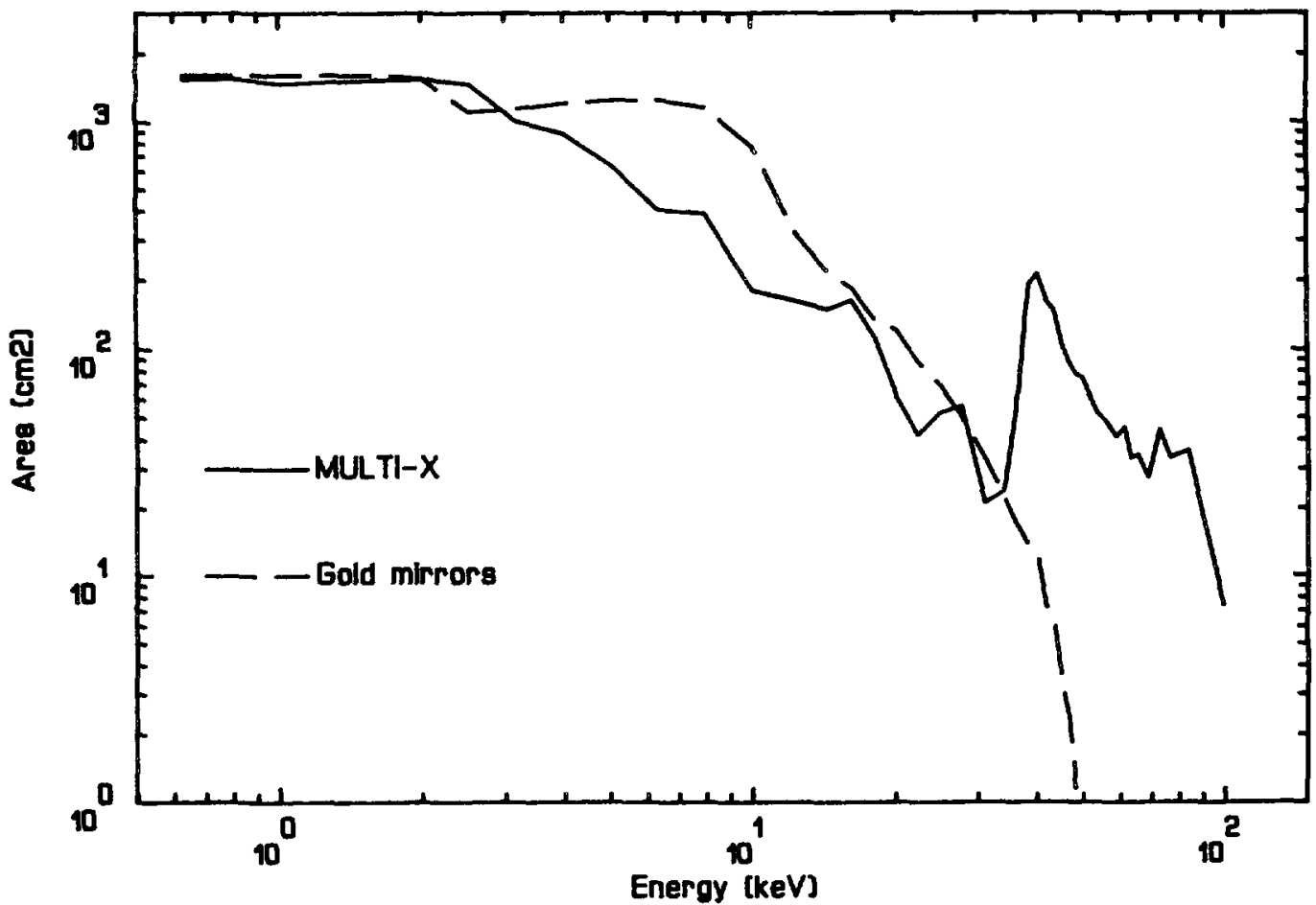

Fig. 4. - Effective area versus energy for the telescope defined in table I (MULTI-X). Dashed line is using a conventional Au coating and the solid line is using a graded perod multilayer coating.

Table II. - Parameters of the multifocus KirkPatrick-Baez telescope in figure 5.

Telescope length

Front-end dimension

Module size

Number of modules

Reflectors pr. module

Reflector length

Reflector thickness

Min. refl. spacing

Total surface area

$330 \mathrm{~cm}$
$48 \mathrm{~cm} \times 48 \mathrm{~cm}$
$6 \mathrm{~cm} \times 6 \mathrm{~cm}$
64
80
$45 \mathrm{~cm}$
$0.25 \mathrm{~mm}$
$0.5 \mathrm{~mm}$
$138 \mathrm{~m}^{2}$

reflectivity studies have been performed at X-ray energies from $8 \mathrm{keV}$ to $100 \mathrm{keV}$ using both laboratory and synchrotron radiation sources [30]. The conclusion is that highly perfect X-ray supermirrors can be produced with interfacial roughnesses of $\sim 5 \AA$ for $\mathrm{Ni} / \mathrm{C}$ structures and approximately the same for W/Si [30]. In the near future $\mathrm{W} / \mathrm{B}_{4} \mathrm{C}$ supermirror coatings will be produced. This material combination is known to be superior to both $\mathrm{Ni} / \mathrm{C}$ and $\mathrm{W} / \mathrm{Si}$ and can have an interfacial roughnesses as small as $3 \AA[31,32]$. Using $W$ as the heavy element works 
Table III. - Telescope parameters for the multifocus conical telescope in figure 5.

\begin{tabular}{|lc|}
\hline Total length & $330 \mathrm{~cm}$ \\
Front-end dimension & $48 \mathrm{~cm} \times 48 \mathrm{~cm}$ \\
Oter radius of one module & $6 \mathrm{~cm}$ \\
Inner radius of one module & $1.5 \mathrm{~cm}$ \\
Number of modules & 16 \\
Reflectors pr. module & 31 \\
Reflector length & $45 \mathrm{~cm}$ \\
Reflector thickness & $0.25 \mathrm{~mm}$ \\
Min. refl. spacing & $0.5 \mathrm{~mm}$ \\
\hline
\end{tabular}

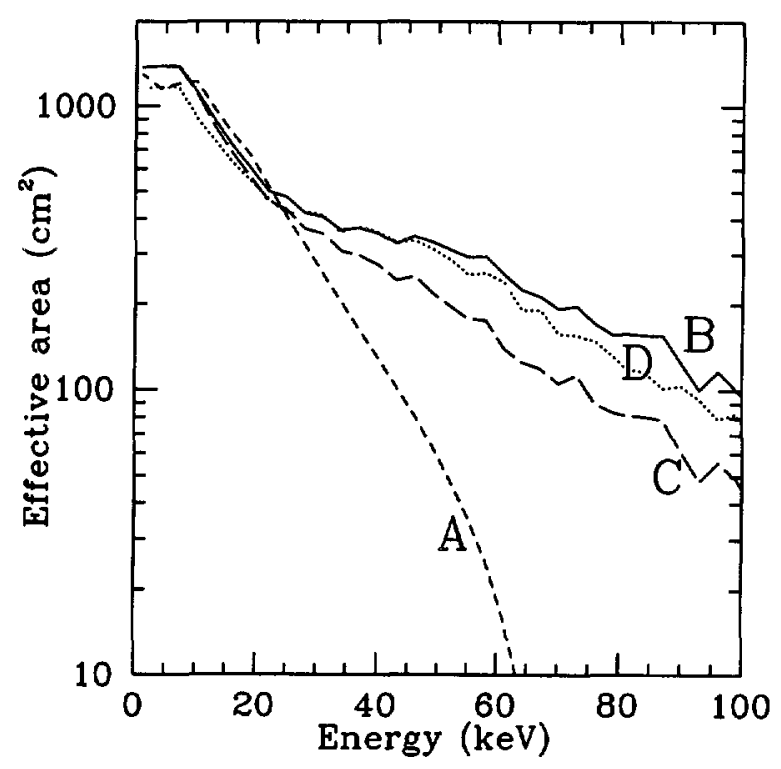

Fig. 5. - Effective area of modular conical telescope (B : solid line), modular KırkPatrick-Baez telescope (D: dotted line) and a modular conical telescope (C: long dashed line) with interfacial roughness of $4 \AA$ compared to a Au coated KirkPatrick-Baez telescope (A : short dashed line). Tables II and III give the telescope configurations.

only for energies below the $\mathrm{K}$ absorption edge $(69.5 \mathrm{keV})$. There is, however, much exciting astrophysics to be obtained up to this energy. There is still work to be done in optimizing the graded period multilayer coatings for X-ray telescopes. It is essential to evaluate what could be obtained with focal lengths of 10-12 m. An array of telescopes of this kind could be the basis of a major ESA cornerstone mission after the year 2000 [32]. Even larger models could be part of a lunar observatory. Finally the X-ray supermirrors are equally useful for single reflection concentrators and such a concentrator, could be flown on a Balloon flight. 


\section{The ultra high resolution asymmetric crystal spectrometer-UHAXS.}

The development of spectrometers with energy resolutions of $E / \Delta E>10^{4}$ is essential for the study of Doppler broadening, large scale collective motions and non-equilibrium phenomena in X-ray space research. In the case of solar studies, this energy resolution should be combined with subarc-second imaging as noted by Walker et al. [34]. They have proposed a design for this type of instrument which is based on two serial reflections $((1,-1)$ geometry $)$ in highly perfect quartz crystals placed in front of an imaging telescope (a double crystal objective crystal spectrometer). The spectrometer will be used to study of FeK-emission line spectra from the Solar corona. Alternatively, Christensen and Schnopper [35] have proposed the use of an asymmetric reflection in a large perfect crystal of $\mathrm{Si}$ or $\mathrm{Ge}$ which provides the possibility of tuning the energy resolution by controlling the asymmetry cut while maintaining an efficient use of the crystal surface and minimizing the requirements on the subsequent imaging optics (Fig. 6). The configuration is a specific version of the objective crystal spectrometer previously described and its ability to make spectrally resolved images of extended sources has already been demonstrated in reference [14]. What is required is to specify the parameters of the asymmetric crystal reflection and the imaging properties of the telescope. For an asymmetric reflection in a perfect crystal where the angle between the surface and the reflecting plane is $\phi$ and where the rays are incident on the non-grazing side, the rocking curve width $W_{\mathrm{RC}}(\phi)$ is [36] :

$$
W_{\mathrm{RC}}(\phi)=W_{\mathrm{RC}}(0) \cdot \sqrt{\sin \left(\theta_{\mathrm{B}}-\phi\right) / \sin \left(\theta+\phi_{\mathrm{B}}\right)} .
$$

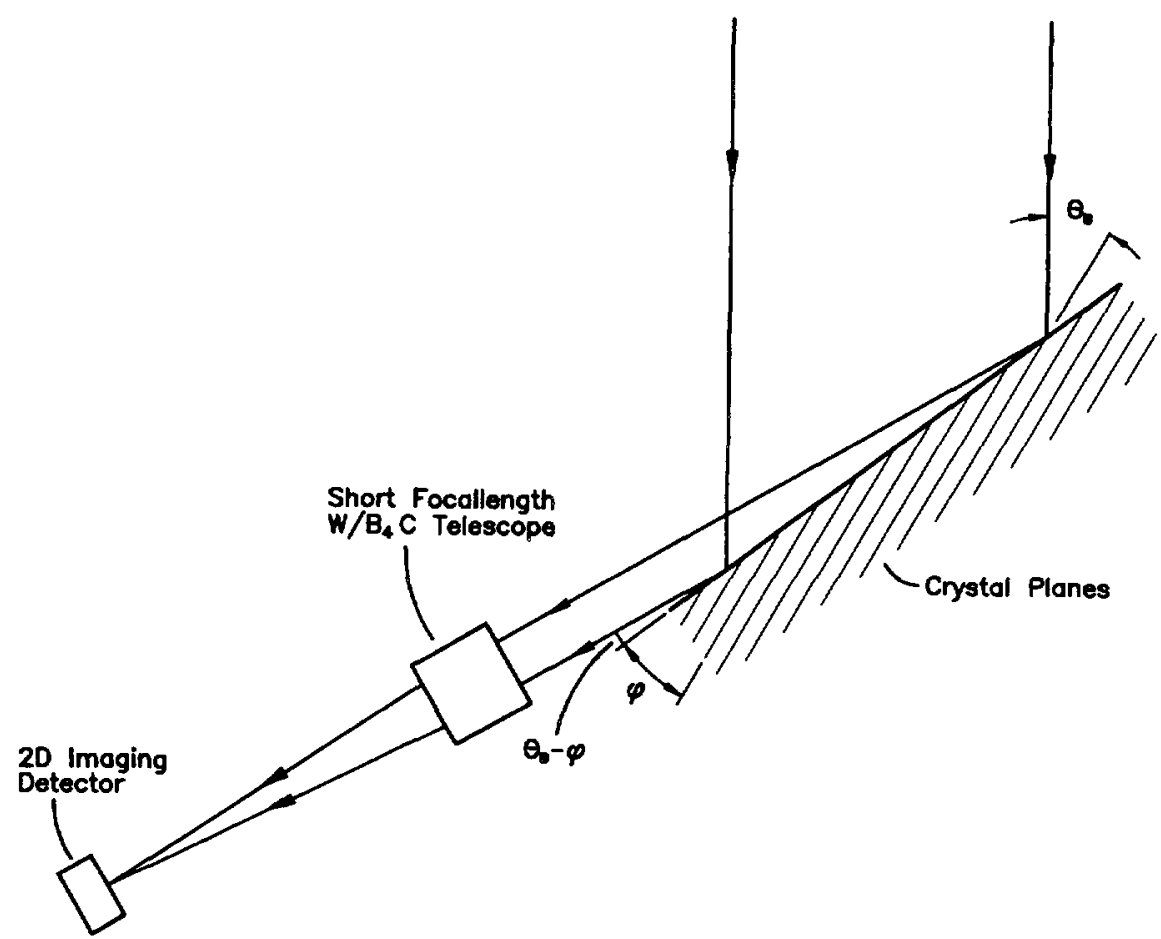

Fig. 6. - A schematic drawing of the asymmetric crystal spectrometer in front of a small, short focal length, multilayer coated Wolter 1 telescope. $\theta_{\mathrm{B}}$ and $\phi$ are defined in the figure. 
Where $\theta_{\mathrm{B}}$ and $\phi$ are defined in figure 6 and $W_{\mathrm{RC}}(0)$ is the rocking curve width of a crystal where $\phi$ is 0 meaning that the reflecting planes are parallel to the surface i.e., a symmetric reflection. Equation (1) allows one to tailor the resolution to be better than what could be obtained with a symmetric reflection and, at the same time compress the beam significantly. This means that the subsequent optics can be much more compact than would be necessary if a symmetric reflection was used. As a specific design either $\mathrm{Si}(220)$ or $\mathrm{Ge}(220)$ can be used for the study of the He-like Fe emission line complex near $6.665 \mathrm{keV}$. A reasonable compromise between energy resolution and beam compression is to choose $\phi$ to be $\sim 23^{\circ}$ which gives an energy resolution of $E / \Delta E$ of $4 \times 10^{4}$ for $\mathrm{Si}(220)$ and $2.6 \times 10^{4}$ for $\mathrm{Ge}(220)$ at $6.665 \mathrm{keV}$. The beam will be compressed by a factor of $\sin \left(\theta_{\mathrm{B}}+\phi\right) / \sin \left(\theta_{\mathrm{B}}-\phi\right)=7.6$ for $\phi=23^{\circ}$ and $\theta_{\mathrm{B}}=29^{\circ}$ (He-like Fe emission). It is assumed that crystals of dimensions $200 \times 100 \mathrm{~mm}^{2}$ can be readily obtained. Beam expansions of more than a factor of 100 for this type of crystals [37] have recently been demonstrated. If the $200 \mathrm{~mm}$ dimension is in the dispersive direction then the beam, after reflection in the crystal, is only $26 \times 100 \mathrm{~mm}^{2}$ According to equation 1 , the energy resolution changes with $\theta_{\mathrm{B}}$, but for $\operatorname{Si}(220)$ this is only from $\sim 5.6 \times 10^{4}$ to $\sim 6.3 \times 10^{4}$ over the wavelength range covered by the He-like $\mathrm{Fe}$ line complex. The reflectivity of the $\mathrm{Si}(220)$ and the $\mathrm{Ge}(220)$ reflections at $6.665 \mathrm{keV}$ are $83 \%$ and $79 \%$. respectively. With a $\mathrm{W} / \mathrm{B}_{4} \mathrm{C}$ multilayer coating, an imaging Wolter 1 telescope can have a short focal length, $1 \mathrm{~m}$ or less. The $\mathrm{W} / \mathrm{B}_{4} \mathrm{C}$ coating for this energy is extremely simple and consists of a maximum of 20 periods with an optimized ratio of $W$ to $B_{4} C$ [32] that gives reflectivities of up to $60 \%$. The grazing angle range for a $0.5 \mathrm{~m}$ focal length telescope behind a $200 \times 100 \mathrm{~mm}^{2}$ crystal is from $0.28^{\circ}$ to $1.43^{\circ}$. This requires that the smallest period of the $\mathrm{W} / \mathrm{B}_{4} \mathbf{C}$ coating is $37 \AA$. The same type of coating was also suggested by Walker et al. for the telescope in their double crystal objective crystal spectrometer [34]. The concept of an asymmetric reflection is also useful for ultra high resolution spectroscopy of cosmic point sources. In this case the telescope would be replaced with a simple one-reflection, high throughput, multilayer coated concentrator. It is estimated that one unit, consisting of a $200 \times 100 \mathrm{~mm}^{2}$ crystal followed by a tightly nested concentrator, could have an effective area of $\sim 55 \mathrm{~cm}^{2}$ per energy resolution bin. Tens of these units can be manufactured inexpensively allowing a total collecting area of several thousand $\mathrm{cm}^{2}$ to be obtained.

\section{A high throughput multilayer telescope for high resolution Fe line spectroscopy.}

With the development of microcalorimeters with demonstrated energy resolutions of 15 $25 \mathrm{eV}[38,39]$ at 6-7 keV, the need arises for an imaging, high throughput, telescope with moderate spatial resolution (1-2 arcmin) that, in combination with the microcalorimeters can provide high resolution spectroscopy of cosmic X-ray sources up to and including the important Fe emission line complex. Such a mission (AXAF-S) has been proposed as a follow up to the planned AXAF-I mission [40]. A small focal length telescope with moderate imaging capability of 1-2 arcmin would make it much easier to build an array of calorimeters. This can be accomplished by combining Au and multilayer coatings optimized for reflectivity at 6 $7 \mathrm{keV}$. Joensen et al. have given specific design examples of such telescopes [32]. To illustrate the capabilities of such a small payload we show in figure 7 the effective area of a graded period multilayer telescope with a focal length of $3 \mathrm{~m}$ and a diameter of $50 \mathrm{~cm}$. This telescope design is an ideal solution for small missions geared to high-throughput, broadband spectroscopy for large sounding rockets and small explorers. Since an observation of the supernova remnant CAS A would be an excellent demonstration of the plasma diagnostic capability of the micro-calorimeter, we show in figure 8 the expected spectrum for a $1000 \mathrm{~s}$ observation from CAS A using the graded period multilayer telescope. This simulation uses 


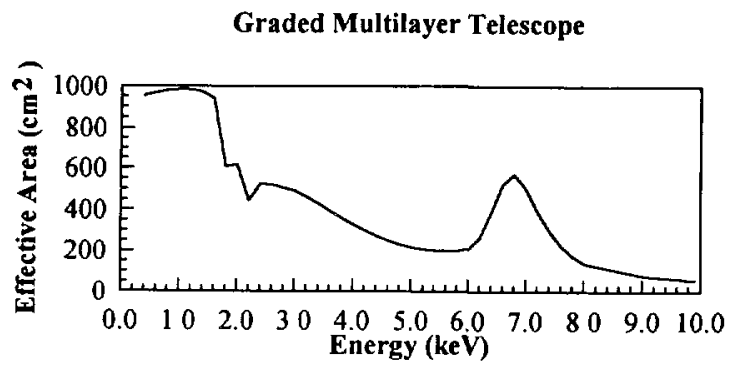

Fig. 7. - The effective area of a graded period multılayer telescope with focal length of 3 meters and diameter of $50 \mathrm{~cm}$.

\section{A Simulated CAS-A Spectrum for 1000 Seconds of Observation Time}
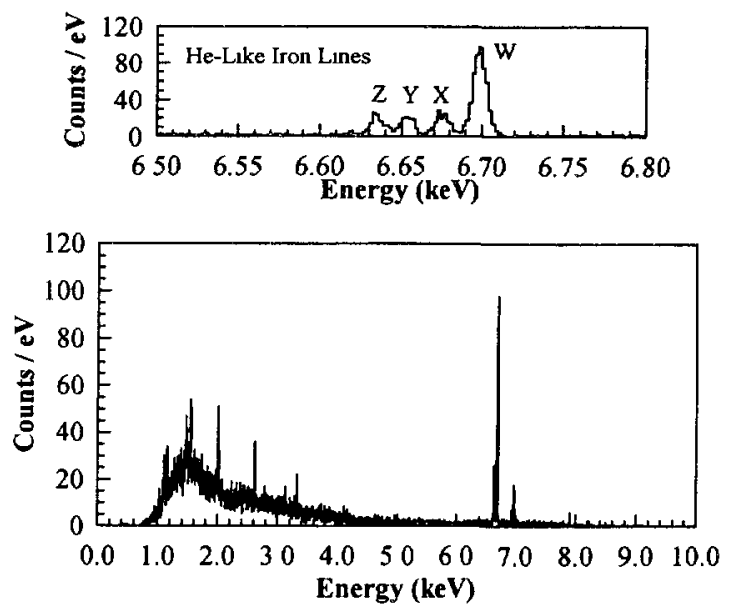

Fig. 8. - The simulated spectrum from the CAS-A supernova remnant that could be obtaned with a microcalorimeter array at the focus of the graded period multulayer telescope defined by figure 7 (bottom). The energy resolution is $10 \mathrm{eV}$ and Poisson noise has been added. An expended view of the energy spectrum near $6.7 \mathrm{keV}$ depicts the He-lıke iron emission lines, $n, r, \quad$ and $z$ (top).

the coronal emission code of Raymond and Smith [41] normalized to the source strength as measured by the gas scintillation propertional counter aboard tenma [42]; Poisson noise has also been included. The line to continuum ratios are excellent. The ability to resolve the Helike iron lines (FeXXV) are also shown in figure 8 . The resonance, intercombination and forbidden lines are designated as $x, \lambda$ and $y$, and $z$, respectively. The relative strengths of these emission lines make it possible to diagnose the electron temperature and density of the emitting stellar plasma.

\section{Summarv.}

A number of novel potential applications of nanostructures in X-ray space research have been described. These include broad band spectroscopy in the 10-100 keV band using graded period multilayer coatings, relatively low resolution spectroscopy in the soft $X$-ray range using multilayers on Si crystals, high and ultra high resolution spectroscopy at FeK line energies 
using the combination of either microcalorimeters or asymmetric crystal reflections together with short focal length multilayer telescopes.

\section{Acknowledgments.}

Discussions with Hans Ulrik Nørgaard-Nielsen and Carl Budtz-Jørgensen at the Danish Space Research Institute is gratefully acknowledged.

\section{References}

[1] Culhane J. L., Cabriel A. H., Acton L. W., Rapley C. G., Phillips K. J., Wolfson C. J., Antonucc1 E., Bentley R. D., Catura R. C., Jordan C., Kayat M. A., Kent B. J., Leibacher J. W., Parmar A. N., Sherman J. C., Springer L. A., Strong K. T., Veck N. J., X-ray spectra of solar flares obtained with a high-resolution bent crystal spectrometer, Ap. J. Lett. 244 (1981) L 141-L 145.

[2] Winkler P. F., Canizares C. R., Clark G. W., Markert T. H., Kalata K., Schnopper H. W., A survey of X-ray line emission from the supernova remnant puppis A, Ap. J. Lett. 246 (1981) L 27.

[3] Giacconi R., Branduardi G.. Briel U., Epstein A., Fabricant D., Feigelson E.. Forman W., Gorenstein P., Grindlay J., Gursky H., Harnden F. R. Jr., Henry J. P., Jones C., Kellogg E., Koch D., Murray S., Schreier E., Seward F., Tananbaum H., Topka K., Van Speybroeck L.. Holt S. S., Becker R. H., Boldt E. A., Serlemitsos P. J., Clark G., Canizares C., Markert T., Novick R., Helfand D., Long K., The Einstein (HEAO 2) X-ray observatory, Ap. J. 230 (1979) 540-550.

[4] Seward F. D., Chlebowski T., Delvaille J. P., Henry J. P., Kahn S. M., van Speybroeck L., Dijkstra J., Brinkman A. C., Heise J., Mewe R., Schrijver J., Calibration and efficiency of the Einstein objective grating spectrometer, Appl. Opt. 21 (1982) 2012.

[5] den Boggende A. J. F., de Korte P. A. J., Videler P. H.. Brinkman A. C., Kahn S. M., Craig W. W., Hailey C. J., Neviére M., Efficiency of X-ray reflection gratings, SPIE 982 (1988) 283.

[6] Brinkman A. C., van Rooijen J. J., Bleeker J. A. M., Dijkstra J. H., Heise J., de Korte P. A. J., Mewe R., Paerels F., Astro. Lett. Commun. 26 (1987) 73.

[7] Schattenburg M. L., Canizares C. R., Dewey D., Levine A. M., Markert T. H., Smith H. I., Transmission Grating Spectroscopy and the Advanced X-ray Astrophysics Facility (AXAF), SPIE 982 (1988) 210.

[8] Underwood J. H., Bruner M. E., Haisch B. M., Brown W. A., Acton L. W., X-ray Photographs of a Solar Active Region with a Multilayer Telescope at Normal Incidence, Science 238 (1987) 61 .

[9] Spiller E., Stearns D., Krumrey M., Multilayer X-ray mirrors : Interfacial roughness, scattering, and image quality, J. Appl. Phys. 74 (1993) 107.

[10] Lindblom J. F., Walker A. B. C., Hoover R. B., Barbee T. W., van Pattern R. A., Gill J. P., Soft $\mathrm{X}$-ray/Extreme Ultraviolet Images of the Solar Atmosphere with Normal Incidence Multilayer Optics, SPIE 982 (1988) 316.

[11] Priedhorsky W. C., Bloch J. J., Smith B. W., Strobel K., Ulibarri M., Chavez J., Evans E., Siegmund O. H. W., Marshall H.. Vallerga J., Vedder P., ALEXIS : An Ultrasoft X-ray Monitor Experiment Using Miniature Satellite Technology, SPIE 982 (1988) 188.

[12] Courvoisier T. J.-L., Orr A., Bühler P., Zehnder A., Henneck R., Stauffacher F., Biakhowski J., Schlumpf N., Schoeps W., Mchedlishvili A., Sunyaev R., Arefev V.. Yascovich A., Babalyan G., Pavlinsky M., Delaboudiniere J. P., Carone T., Siegmund O., Warren J., Leahy D., Salaschenko N., Platonov J., EUVITA - an Extreme UV Imaging Telescope Array with Spectral Capability, To be published in Exp. Astrophys. 
[13] Angel R. P.. Weisskopf M., Astron. J. 75 (1970).

[14] Schnopper H. W., Byrnak B. P., Bragg imaging of extended cosmic X-ray sources: The objective crystal spectrometer, Appl. Opt. 26 (1987).

[15] Christensen F. E.. Byrnak B. P., Hornstrup A., Shou-hua Z., Schnopper H. W., Objective Crystal Spectrometer (OXS) for the Spectrum-X- $y$ satellite, SPIE 1344 (1990) 14.

[16] Christensen F. E., Rasmussen I., Schnopper H. W., Wiebicke H.-J., Halm I., Geppert U., Borozdin K., Qualification study of LiF flight crystals for the objective crystal spectrometer on the Spectrum-X- $y$ satellite, SPIE 1743 (1992) 181.

[17] Halm I., Wiebicke H.-J.. Geppert U., Christensen F. E., Abdali S., Schnopper H. W., Analysis of rocking curve measurements of $\mathrm{LiF}$ flight crystals for the objective crystal spectrometer on SPECTRUM-X- $\gamma$, SPIE 2006 (1993).

[18] Spiller E.. Monograph on Soft X-ray Optics and Multilayer Structures, to be published by SPIE.

[19] Puik E. J., Ph. D. thesis, Multilayer X-ray coatings : Progress in deposition, Characterization and application (1990).

[20] Louis E., Voorma H.-J., Koster N. B., Shmaenok L., Bijkerk F., Schlatmann R., Verhoeven J., Platonov Yu. Ya., van Dorssen G. E., Padmore H. A., Enhancement of reflectivity of multilayer mirrors for soft $\mathrm{X}$-ray projection lithography by temperature optimization and ion bombardement. Accepted for publication in a special issue of Microelectronic Engineering containing the proceedings of the ME-93 in Maastricht.

[21] Fraser G. W., Brunton A. N., Lees J. E., Emberson D. L., Nucl. Inst. Meth. A 334 (1993) 404. Fraser G. W.. Production of quas1-parallel X-ray beams using microchannel plate X-ray lenses, Nucl. Inst. Meth. A 344 (1993) 579.

[22] Gorenstein P., Non-Traditional Grazing Incidence Optics for X-ray Astronomy, SPIE 1736 (1992) 201.

[23] Schnopper H. W., Bragg reflection concentrators for hard X-ray astronomy, Appl. Opt. 20 (1981) 1089.

[24] De Chiara P., Frontera F., Bragg diffraction technique for the concentration of hard X-rays for space astronomy, Appl. Opt. 31 (1992) 1361.

[25] Christensen F. E., Hornstrup A., Westergaard N. J., Schnopper H. W., Wood J., Parker K., A graded d-spacing multilayer telescope for high energy X-ray Astronomy, SPIE 1546 (1991) 160.

[26] Wolter H., Ann. Physik NY 10 (1952) 94 ;

Wolter H., Ann. Physik, NY 10 (1952) 286.

[27] Mezeı F., Common. Phys. 1 (1976) 81 ;

Schelten J., Milka K., Calculated reflectivities of super-mirrors, Nucl. Inst. Meth. 160 (1979) 287 ; Hayter J. B., Mook H. A., J. Appl. Cryst. 22 (1989) 35.

[28] Westergaard N. J., Byrnak B. P., Christensen F. E., GrundsøE P., Hornstrup A., Henrichsen S., Henriksen U., Jespersen E., Noergaard-Nielsen H. U., Polny J., Schnopper H. W., Ørup P., Status of the development of a thin-foil, high-throughput $\mathrm{X}$-ray telescope for the Soviet Spectrum X- $\gamma$ mission, Opt. Eng. 29 (1990) 6.

[29] Joensen K. D., Christensen F. E., Schnopper H. W.. Gorenstein P., Susini J., Høghøj P., Hustacke R., Wood J., Parker K., Medium-sized grazing incidence high-energy X-ray Telescopes employing continuously graded multilayers, SPIE 1736 (1992) 239.

[30] Joensen K. D., Høghøj P., Christensen F. E., Gorensteın P., Susıni J., Ziegler E., Wood J., Multilayered supermirror structures for hard X-ray synchrotron and astrophysics instrumentation, SPIE 2011 (1993).

[31] Christensen F. E., Shou-hua Z., Hornstrup A., Schnopper H. W., Plag P., Wood J., X-ray Study of State-of-the-Art Small d-spacing W/B $\mathrm{B}_{4} \mathrm{C}$ Multilayers, J. X-ray Sct. Tech. 3 (1991) 1.

[32] Joensen K. D., Gorenstein P., Christensen F. E., Gutman G., Grazing Incidence Fe-line telescopes using $\mathrm{W} / \mathrm{B}_{+} \mathrm{C}$ multilayers, To be submitted to Appl Phys.

[33] Turner M., Cole R. E., Fraser G. W., Willingale R., Christensen F. E., Schnopper H. W., Lund N.. Frontera F., Gnedin Yu. N., Cruise A. M., A Hard X-ray Sky Survey, Spectroscopy, and Polarimetry Mission (HXT), Response to call for Mission Concepts from ESA. 
[34] Walker A. B. C., Willis T. D., Hoover R. B., The Objectıve Double Crystal Spectrometer, SPIE 1546 (1991) 461.

[35] Christensen F. E., Schnopper H. W., Calls for Proposals and ideas for Columbus Precursor Flights (1991).

[36] Zachariassen H. W., Theory of X-ray diffraction in crystals, Dover, New York (1935).

[37] Christensen F. E., Hornstrup A., Frederiksen P., Abdalı S., Grundsøe P., Schnopper H. W., Lewis R., Hall C. H., Borozdin K., The Expanded Beam X-ray Optics Calibration Facility at the Daresbury Synchrotron, SPIE 2011 (1993).

[38] Juda M., Cui W., McCammon D., Morgenthaler J., Sanders III W. T., Zhang J., Kelley R. L., Madejskı G. M., Moseley S. H., Szymkowiak A. E., Thermal detectors for X-ray astronomy: Current performance and Limitations, SPIE 1743 (1992) 398.

[39] Silver E. H., LeGros M., Labov S., Goulding F., Madden N., Landis D., Beeman J., Microcalorimeters for High Resolution X-ray Spectroscopy of Laboratory and Astrophysical Plasmas, Proposal submitted to the High Energy Astrophysics X-ray Astronomy Research and Analysis Program Response to NRA-93-OSS-3 (1993).

[40] Weiskopf M., AXAF VETA test - an overview, SPIE 1742 (1992).

[41] Raymond J., Smıth B., Soft X-ray Spectrum of a Hot Plasma, Ap. J Suppl. 35 (1977) 419.

[42] Tsunemi H., Yamashita K., Masa1 K., Hayakawa S., Koyama K., X-ray Spectra of the Cassiopeia A and Tycho Supernova Remnants and their element Abundances, Ap J. 306 (1986) 248. 